\title{
Resection Angulation Osteotomy in Treatment of Post- septic Ankylosis of the Hip
}

\author{
DG Nabi, MS, TS Rashid, MS, KK Ahmed, MS \\ Department of Orthopaedics, Government Medical College, Srinagar, India
}

\begin{abstract}
This study evaluated the results of the resection angulation osteotomy in post-septic ankylosis of hip. We performed the procedure in 19 patients, with average age of 26 years (range, 21-32 years) and followed patients postoperatively for an average of 43 months (range 20-65 months). The osteotomy united in all patients with an average union time of 16 weeks (range 12-22 weeks). Average painless movement postoperatively was: flexion, 114 degrees; extension, 18 degrees; abduction, 35 degrees; adduction , 21 degrees; external rotation, 23 degrees; and internal rotation, 22 degrees. All the patients except one were able to sit crosslegged on the floor, offer prayers and use squat-type toilets. We conclude that this procedure is a good treatment option in patients with post-septic ankylosis of the hip, who need an alternative to more sophisticated procedures like total hip arthroplasty.
\end{abstract}

Key Words:

Ankylosed hip; septic arthritis; Osteotomy.

\section{INTRODUCTION}

Loss of the femoral head and neck as a result of disease, trauma or surgical procedure is usually regarded as failure, and most orthopaedic surgeons are reluctant to accept this condition as a satisfactory end result of treatment. Patients with this defect, however, often consider the functional result highly satisfactory because of relatively painless mobility ${ }^{1}$. The obvious disadvantage of the result is the instability of the hip and shortening of the limb ${ }^{2}$. Angulation osteotomy undoubtedly increases the stability of the hip ${ }^{3}$, also positions abductors of the hip at a better angle leading to an improvement in gait.

Movements of the hip joints are of paramount importance for squatting, sitting cross-legged, offering prayers, using squattype toilet and for sexual intercourse. Although hip ankylosis may be well tolerated in Western societies, ankylosis is usually not acceptable to Asians. With the advent of more sophisticated procedures, resection of head and neck is now seldom used as a primary form of treatment ${ }^{3}$. Total hip arthroplasty is effective in alleviating pain, and provides a mobile hip with great stability, but it is not a panacea and has its own limitations and contraindications. Young age, risk of infection (especially with previous history of infection), and sometimes the necessity for revision demand the availability of an alternative method of treatment. It may be therefore be worthwhile to try resection of head and neck combined with pelvic support osteotomy in selected cases to restore the movements with added advantage of stability at the hip.

There are some published papers in literature that discuss the results of Girdlestone excision with or without pelvic support osteotomy following failed surgical procedures of the hip ${ }^{4,5,6}$. We report herein the functional results of resection angulation osteotomy in patients with post-septic ankylosis of the hip.

\section{MATERIALS AND METHODS}

From March 2002 to December 2005, 19 patients whose hips were fused in non-anatomical position secondary to septic arthritis [Fig 1 and 3] were treated with resection angulation osteotomy and followed for an average period of 43 months (range, 20-65 months). The primary indications for surgery were stiffness and deformities of the hip. Patients included in the study were those who desired to sit on the floor cross legged, kneel while offering prayers, use squat-type toilets and who requested an alternative to total hip arthroplasty. Ages range for patients were between 21-32 years (average age, 26 y); 12 were male and seven were female patients, and the right hip was affected in 11 and left in 8 patients. The average time period between primary disease (septic arthritis) and resection angulation osteotomy was 18.5 years (average, 15-25 y).

Surgery was performed with the patient in supine position, under epidural or spinal anaesthesia using the Watson-Jones lateral approach, excision of the femoral head and neck and a pelvic support osteotomy were carried out as a single procedure. The osteotomy was carried out below the lesser trochanter at the level of the ischial tuberosity. Following excision of the femoral head and neck, a tongue of bone was outlined with multiple drill holes on the lateral part of the distal fragment. The bone was divided around its 


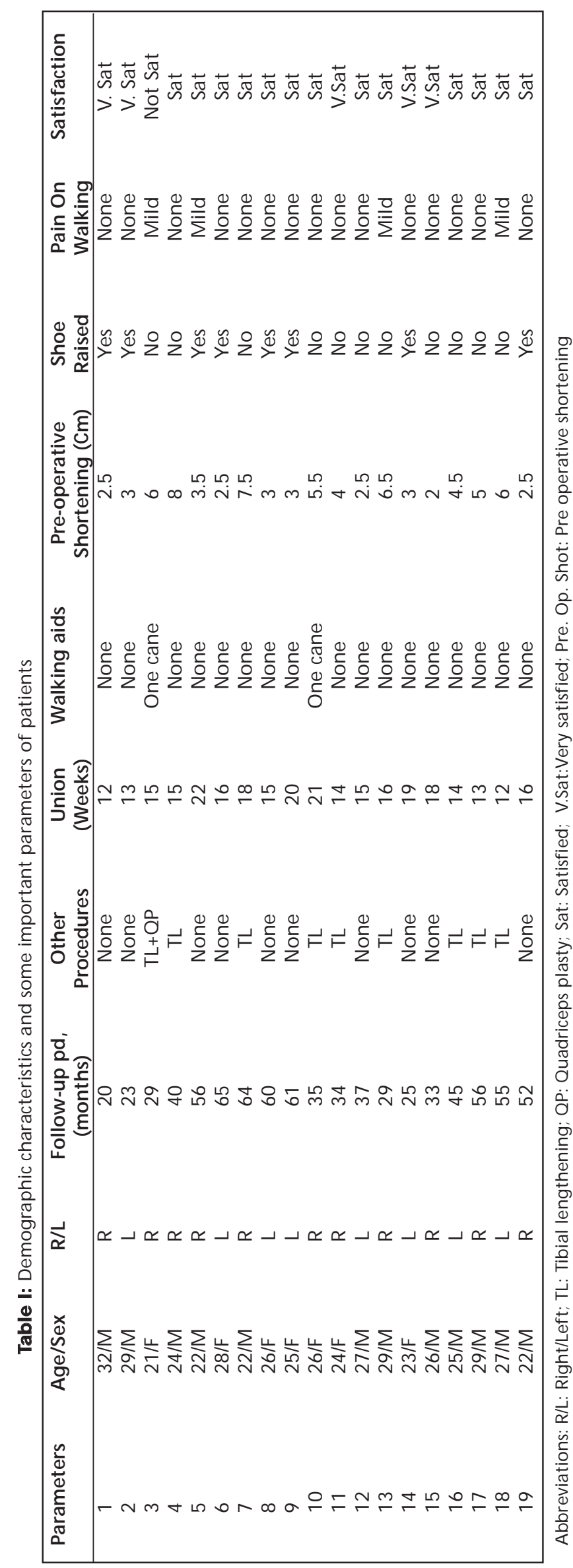




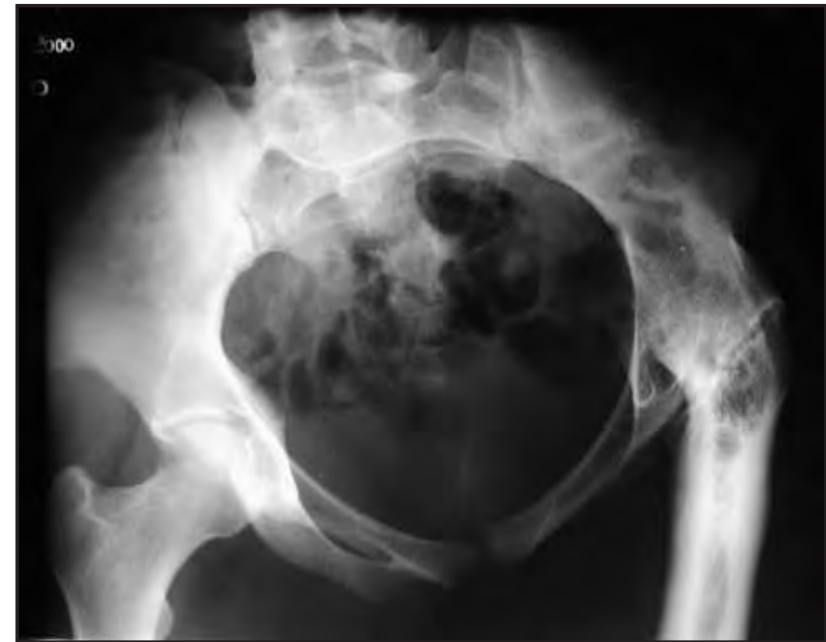

Fig. 1: Ankylosed left hip in non-anatomical position.

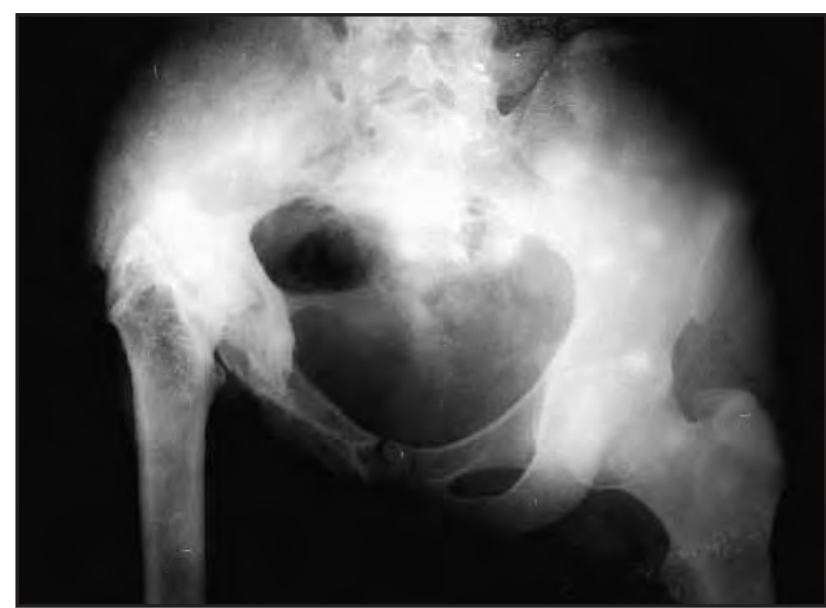

Fig. 3: Ankylosed Right hip in non-anatomical position.

circumference at the base of tongue. The distal fragment was abducted after impaction of the tongue in the proximal fragment. The osteotomy was angulated medially beneath the acetabulum and fixed by a plate bent to the desired angle.

Postoperative skeletal traction was applied through the upper tibial pin. Range of motion exercises of the hip and knee were started at 2 weeks post surgery while the patients were still in traction. Patients ambulated at six weeks with the help of a walker or crutches with strict instructions not to bear weight. Weight bearing was permitted only after osteotomy union. Secondarily, tibial lengthening by distraction osteogenesis using Ilizarov ring fixation, was performed in 9 patients after consolidation of the osteotomy. One patient needed quadriceps plasty for stiffness of the knee [Table I].

\section{RESULTS}

The results were assessed considering functional status in terms of painless motion at the hip, painless ambulation,

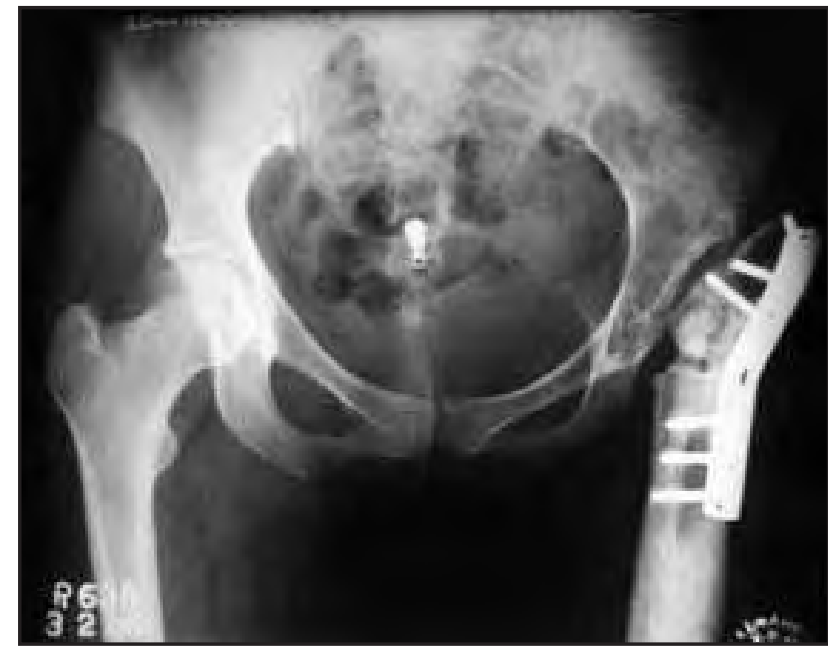

Fig. 2: Resection angulation osteotomy.

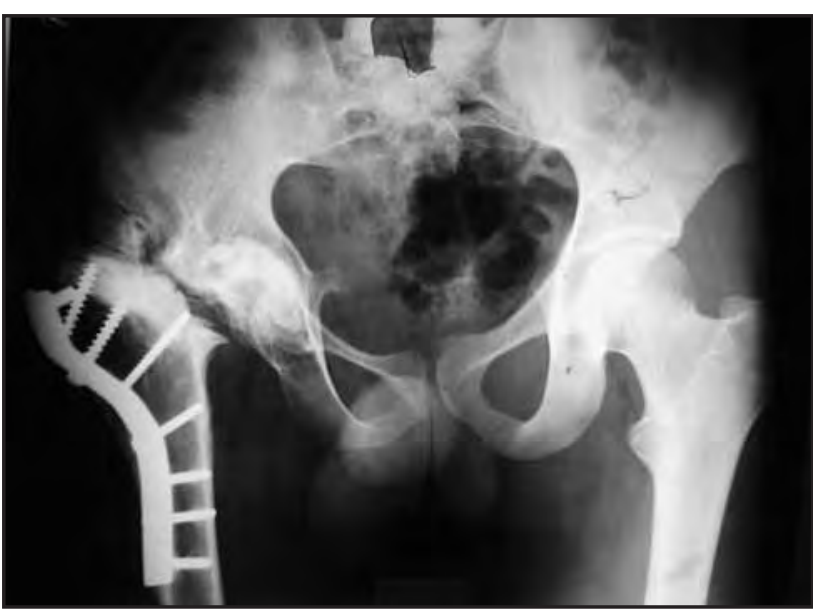

Fig. 4: Postoperative photograph of resection angulation osteotomy.

ambulation with or without support and over all satisfaction with the procedure expressed by the patient. Osteotomy union occurred in all studied patients with the average time for union being 16 weeks (range, 12-22 weeks) [Fig 2 and 4]. All patients had reasonably painless motion at the hip. Range of motion was: flexion of $100-130^{\circ}$ (average- $114^{\circ}$ ); extension of $10-30^{\circ}$ (average- $18^{\circ}$ ); abduction of $25-40^{\circ}$ (average-35 degrees); adduction of $20-30^{\circ}$ (average- $21^{\circ}$ ); external rotation of $20-30^{\circ}$ (average- $23^{\circ}$ ); and internal rotation of 15 $30^{\circ}$ (average- $22^{\circ}$ ) were achieved. None of the patients complained of moderate to severe pain during walking. Seventeen out of nineteen needed no support for ambulation at the final follow up. Only one was unable to sit crosslegged on floor, offer prayers and use squat- type toilets, and this was due to somewhat limited flexion at the knee. There were no complications associated with the resection angulation osteotomy except one case of transitory foot drop and knee valgus in two patients, all associated with tibial lengthening. Eighteen out of nineteen were able to perform the daily activities of life independently and were satisfied with the surgical results [Table I]. 


\section{DISCUSSION}

Movement at the hip joint is of great significance for walking and other activities of daily life. Squatting posture and sitting cross-legged practiced is important in Asian and Indian culture and requires good range of movements at the hip joints. Ankylosis of the hip results in malpositioning that can lead to spinal deformity, pelvic obliquity and limb length discrepancy and possible painful degeneration of the spine or the knee.

The Girdlestone technique originally described the resection of head and neck without osteotomy in the treatment of osteoarthritis of the hip ${ }^{7}$. Batchelor added a subtrochanteric osteotomy as a second procedure performed after 5 to 6 weeks ${ }^{8}$, and later Milch described resection angulation osteotomy as a single procedure ${ }^{9}$. On the other hand, Philips reported excellent results in 5 (in whom an angulation osteotomy was added) of his 38 patients and stated that angulation osteotomy increases the stability of the hip and is a worth wile adjunct ${ }^{3}$. With the advent of more sophisticated reconstructive procedures like total hip arthroplasty, resection of the femoral head and neck has come to be regarded as a salvage procedure, to be used only when cups and prosthesis have failed due to infection or technical difficulties. The procedure is seldom used as a primary form of treatment, except for joints with chronic sepsis ${ }^{3}$.

Excision of the femoral head and neck is an excellent method of relieving pain, regardless of the cause and it corrects deformities and restores free movements of the hip ${ }^{4}$. Patients consider the functional result highly satisfactory because of relatively painless mobility ${ }^{1}$. The obvious disadvantage of the procedure is the instability of the hip and shortening of the limb ${ }^{3}$. Angulation osteotomy increases the stability of the hip and puts abductors of the hip at a better angle leading to improvement in gait. The internal fixation of the osteotomy obviates the need for weight relieving calipers. Although the limb is shortened, downward tilting of the pelvis and abduction of the shaft produced apparent lengthening of the extremity.

Our study is unique in that we report the results of resection angulation osteotomy in a series of 19 patients whose hip joints were fused in a non-functional position as sequelae of septic arthritis. The patients were from an indigent background and desired an alternative method to more sophisticated and expensive procedures such as total hip arthroplasty. Rigid internal fixation of the osteotomy permits the early rehabilitation of the patient. All the patients in our series expressed satisfaction with the results obtained and experienced painless ambulation with no support. The most beneficial outcome expressed by the patients was the increased yet painless range of motion. The patients are able to sit cross-legged on floor, offer prayers, have sexual intercourse and use squat-type toilets.

\section{CONCLUSION}

Movements at the hip joint in different directions are of great significance for the activities of daily life,especially in Asian culture. Reconstruction of the hip joint with total hip arthroplasty may not be suitable for indigent population.The gratifying results of femoral head and neck combined with proximal femoral angulation ostyeotomy suggest that the operation can be considered for these types of patients. 


\section{REFERENCES}

1. Murray WR, Lucas DB, Inma VT. Femoral head and neck resection. J Bone Joint Surg Am 1964 46; 6: 1184-97.

2. Laing PG. Anterior angle plates for high femoral osteotomies; J Bone and Joint Surg. 1958 40A; 5: 1158-60.

3. Parr PL Croft C, Enneking WF. Resection of head and neck of the femur with or without angulation osteotomy. A follow up study of thirty eight patients. J Bone Joint Surg 1971 53A; 5: 935-44.

4. Haw CS and Gray DH. Excision arthroplasty of the hip. J Bone Joint Surg 1976; 58B(1): 44-7.

5. Sharma H, De Leeuw J, Rowley DI. Girdlestone resection arthroplasty following failed surgical procedures. International Orthopaedics 2005; 29(2): 92-5.

6. Zabihi T, Kohanim M, Amir-Jahed AK. A modified Girdlestone operation in treatment of complications of fractures of the femoral neck; J Bone and Joint Surg 1973; 55A(1): 129-36.

7. Girdlestone GR. Arthrodesis and other operations for tuberculosis of the hip. In: The Robert Jones Birthday Volume. London: Oxford University Press, 1928: 347.

8. Batchelor JS. Excision of the femoral head and neck in cases of ankylosis and osteoarthritis of the hip. Proc R Soc Med 1945; 28: 689-90.

9. Milch Henry. The resection angulation operation for hip- joint disabilities. J Bone Joint Surg 1955; 37A: 811-27. 